\title{
A self-sustaining nonlinear dynamo process in Keplerian shear flows
}

\author{
F. Rincon $*$ G. I. Ogilvie, and M. R. E. Proctor \\ Department of Applied Mathematics and Theoretical Physics, University of Cambridge, \\ Centre for Mathematical Sciences, Wilberforce Road, Cambridge CB3 OWA, United Kingdom
}

(Dated: October 27, 2018)

\begin{abstract}
A three-dimensional nonlinear dynamo process is identified in rotating plane Couette flow in the Keplerian regime. It is analogous to the hydrodynamic self-sustaining process in non-rotating shear flows and relies on the magneto-rotational instability of a toroidal magnetic field. Steady nonlinear solutions are computed numerically for a wide range of magnetic Reynolds numbers but are restricted to low Reynolds numbers. This process may be important to explain the sustenance of coherent fields and turbulent motions in Keplerian accretion disks, where all its basic ingredients are present.
\end{abstract}

PACS numbers: 47.15.Fe, 47.20.Ft, 47.27.De, 52.30.Cv, 98.62.Mw

The most natural explanation for the efficient outward angular momentum transport inferred from observed accretion luminosities in accretion disks is that these objects are turbulent [1]. In magnetized disks, turbulence is likely triggered by the magneto-rotational instability [MRI, see 2]. When a mean magnetic field with non-zero net flux perpendicular to the disk plane is imposed, the instability takes the form of a two-dimensional channel flow which breaks down into three-dimensional MHD turbulence [3] as a result of secondary Kelvin-Helmholtz instabilities [4]. This simple field configuration, however, may not be relevant to all accretion disks. Recent observations have for instance demonstrated that the magnetic fields in the innermost regions of some disks are probably not created by an external object such as the central accreting body but are intrinsic to the disk [5]. Their precise origin, however, remains largely unknown. Understanding the nonlinear saturation of the MRI in the absence of an externally imposed magnetic field or more generally when there is no net magnetic flux threading through the disk proves to be a complicated task because one needs to explain in that case how the magnetic field, whose presence within the disk is permanently required for the generation of MHD turbulence, can be sustained against dissipation. Local numerical disk simulations in the absence of a net magnetic flux [6, 7] suggest that a dynamo process possibly relying on the MRI of toroidal magnetic fields [8] may be at work, but a detailed theoretical understanding of such a scenario is still lacking.

In this Letter, we report the discovery of self-sustaining dynamo action in magnetized, spanwise rotating plane Couette flow (PCF) in the Keplerian regime (linearly stable from the purely hydrodynamic point of view) characteristic of accretion disks. The phenomenology of this magnetohydrodynamic (MHD) process is analogous to that of the self-sustaining process (SSP) thought to be responsible for the transition to turbulence in hydrodynamic shear flows [9, 10]. It relies on three fundamental physical effects:

1. linear amplification of zero-net-flux toroidal (azimuthal in the accretion disk terminology) magnetic field induced by the distortion of a weak poloidal (radial and vertical) seed field by the background shear (differential rotation);

2. three-dimensional linear instability (MRI) of the toroidal magnetic field ;

3. regeneration of the poloidal field owing to the nonlinear feedback of the MRI modes.

Even though linear processes are essential to the mechanism, either via transient linear growth or linear instability, the whole process is fundamentally nonlinear since the self-sustaining loop cannot be closed without nonlinear feedback. This notably means that there is no kinematic regime for the dynamo, which is therefore subcritical: an initial finite-amplitude, zero-net-flux magnetic field disturbance is required for the dynamo to operate. This contrasts with the externally imposed field problem in which infinitesimal disturbances grow exponentially due to the MRI. To the best of our knowledge, this is the first instance of an explicit nonlinear subcritical dynamo solution. We first describe the three steps of the process, construct steady nonlinear solutions for Keplerian PCF at various magnetic and kinetic Reynolds numbers and finally discuss the relevance of our results to accretion disk theory.

We consider PCF for an incompressible fluid with unit density, constant kinematic viscosity $v$ and magnetic diffusivity $\eta$. The flow is driven by two counter-moving rigid, no-slip, perfectly conducting walls located at $y= \pm d$ and is rotating at a constant rate $\Omega$ along the spanwise (vertical) $z$-axis perpendicular to the linear background shear flow $\boldsymbol{V}_{B}(y)=S y \boldsymbol{e}_{x}$. It is taken to be spatially periodic in both streamwise (toroidal, $x$ ) and spanwise (z) directions, with periods $L_{x}=2 \pi d / \alpha$ and $L_{z}=2 \pi d / \beta$. Using $1 / S$ as a time unit and the channel half-width $d$ as a length unit, we define a Reynolds number $\operatorname{Re}=S d^{2} / v$ and a magnetic Reynolds number $\mathrm{Rm}=S d^{2} / \eta$. This configuration represents an idealized local model of a differentially rotating Keplerian accretion disk provided that the rotation number $R_{\Omega}=-2 \Omega / S$ equals $-4 / 3$ (anticyclonic Rayleighstable rotation). Owing to PCF symmetries, we look for nonlinear three-dimensional steady solutions of the incom- 
pressible MHD equations (Navier-Stokes equation with a Lorentz force and induction equation) for magnetic and velocity field perturbations $\boldsymbol{b}$ and $\boldsymbol{v}$ of Keplerian PCF. We first consider step 1 of the process. We define a streamfunction $\psi(y, z, t)$ and a flux function $\chi(y, z, t)$ to describe the mean poloidal fields $\overline{\boldsymbol{v}}_{\mathrm{p}}=\boldsymbol{\nabla} \times\left(\psi \boldsymbol{e}_{x}\right)$ and $\overline{\boldsymbol{b}}_{\mathrm{p}}=\boldsymbol{\nabla} \times\left(\chi \boldsymbol{e}_{x}\right)$, where overbars stand for $x$-averaging, and denote the $x$-dependent part of the fields by $\boldsymbol{v}^{\prime}$ and $\boldsymbol{b}^{\prime}$. The toroidal and poloidal components of the $x$-averaged induction equation read

$$
\begin{gathered}
\partial_{t} \bar{b}_{x}=\bar{b}_{y}+\boldsymbol{e}_{x} \cdot \overline{\nabla \times(\boldsymbol{v} \times \boldsymbol{b})}+\frac{1}{\mathrm{Rm}} \Delta \bar{b}_{x}, \\
\partial_{t} \chi+\frac{\partial \psi}{\partial z} \frac{\partial \chi}{\partial y}-\frac{\partial \psi}{\partial y} \frac{\partial \chi}{\partial z}=\overline{\left(\boldsymbol{v}^{\prime} \times \boldsymbol{b}^{\prime}\right)} \cdot \boldsymbol{e}_{x}+\frac{1}{\mathrm{Rm}} \Delta \chi
\end{gathered}
$$

Equation (1) has a linear induction source term $\bar{b}_{y}$ due to the presence of the background shear, which makes it possible to generate an $O(1)$ toroidal magnetic field from a weak $O(1 / \mathrm{Rm})$ poloidal field (this results mathematically from the non-normality of the linear operator). This axisymmetric process for two-dimensional, three-component (2D-3C) fields is often referred to as the $\Omega$-effect in dynamo theory and is the MHD analog of the algebraic amplification of streaks (streamwise velocity field) in nonrotating hydrodynamic shear flows, known as the lift-up effect in the fluid dynamics literature [11]. Unless some three-dimensional nonlinear mechanism regenerates the poloidal field, this linear process can however only be transient: for purely two-dimensional configurations, Eq. (2) is a simple advection-diffusion equation with no source term for $\chi$, which must therefore decay resistively on an $O(\mathrm{Rm})$ timescale. This is just a restatement of Cowling's theorem for an axisymmetric ( $x$-independent here) system. To obtain a SSP, we must therefore consider the $x$-dependent, three-dimensional instabilities of the transiently amplified toroidal component of the 2D-3C field (step 2) and their nonlinear feedback in the poloidal equation (step 3). Three-dimensional, nonlinear steady solutions are possible only if the nonlinear interaction term on the r.h.s. of Eq. (2) has the ability to regenerate the original seed poloidal field. For the hydrodynamic SSP, the threedimensional instabilities are inflectional instabilities of the spanwise-modulated finite-amplitude streaks. In the Keplerian MHD problem, a natural instability candidate is a three-dimensional MRI of the $O(1)$ toroidal magnetic field.

We use a three-dimensional nonlinear continuation code based on Newton iteration to test this scenario quantitatively. The code is similar to those used to study hydrodynamic SSPs [10, 12] and has been tested extensively with standard nonlinear problems [13]. Collocation on a GaussLobatto grid is used in $y$ and a Fourier representation (with dealiasing) is used in $x$ and $z$. The Newton solver relies on the linear algebra library LAPACK to solve real systems with $O(20000)$ unknowns. An iterative generalized eigenvalue problem solver from the ARPACK library is used to address $2 \mathrm{D}$ linear stability problems. The convergence of our solutions ( $\sim 10^{-8}$ for energy) has been thoroughly checked by looking at Chebyshev and Fourier spectra and by comparing results obtained at different resolutions.

We adopt a forcing strategy [10], which first consists in artificially forcing nonlinear steady 2D-3C solutions and in computing their three-dimensional instability modes. One must then check that the nonlinear feedback of these modes can take over the forcing to obtain three-dimensional nonlinear unforced solutions. For the MHD problem, an appropriate forcing is a toroidal electromotive force (EMF)

$$
\operatorname{EMF}_{x}(y, z)=\frac{A}{\beta \mathrm{Rm}^{2}} \cos \left(\frac{\pi y}{2}\right) \cos \beta z,
$$

applied to Eq. 22 with $A=O(1)$, which compensates for the resistive decay of an $O(1 / \mathrm{Rm})$ poloidal magnetic field that in turn generates an $O(1)$ toroidal field. Fig. 1 depicts a 2D-3C magnetic field solution of the forced nonlinear MHD equations obtained with our Newton solver.

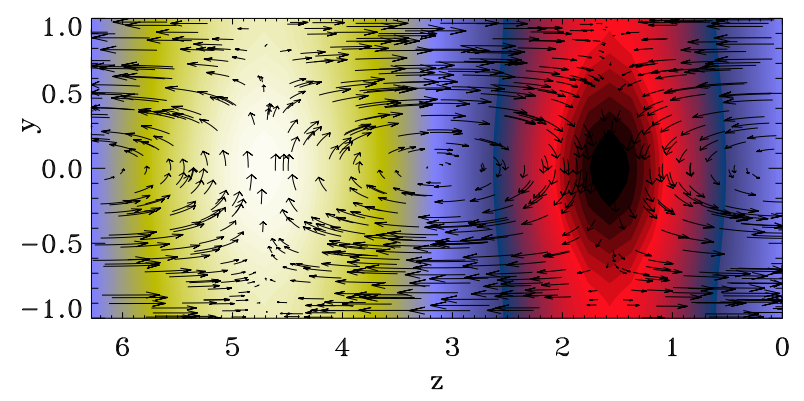

FIG. 1: 2D-3C magnetic configuration induced by the artificial toroidal EMF [3], $\beta=1$ and perfectly conducting boundary conditions. $b_{x}$ is represented on a color scale (black to white from $\max$ to $\max )$ and $\left(b_{y}, b_{z}\right)$ by arrows. $\mathrm{Rm}=750, \mathrm{Re}=10, A=1.5$, $\max \left(b_{x}\right)=1.006$ and $\max \left(b_{y}\right)=0.00195 .\left(N_{y}, N_{z}\right)=(32,32)$.

We then consider the stability of forced 2D-3C MHD flows with a dominant $O(1)$ toroidal magnetic field to infinitesimal perturbations with $\exp (i \alpha x)$ dependence, and compute the associated MRI eigenmodes with our linear eigenvalue solver (the Alfvén continuum is removed here owing to viscous and resistive effects). For the forcing term (3) steady solutions have three symmetries which are used to reduce computational costs: first, they either have reflect $(\mathrm{R}) z \rightarrow-z$ or shift-and-reflect $(\mathrm{SR})(x, z) \rightarrow$ $\left(x+L_{x} / 2,-z\right)$ symmetry which both turn $\left(v_{x}, v_{y}, v_{z}\right)$ into $\left(v_{x}, v_{y},-v_{z}\right)$ and $\left(b_{x}, b_{y}, b_{z}\right)$ into $\left(-b_{x},-b_{y}, b_{z}\right)$. They also have either $z$-shift (S) $z \rightarrow z+L_{z} / 2$ or double-shift (DS) symmetry $(x, z) \rightarrow\left(x+L_{x} / 2, z+L_{z} / 2\right)$ which both turn $(\boldsymbol{v}, \boldsymbol{b})$ into $(\boldsymbol{v},-\boldsymbol{b})$. Finally, owing to the invariance of PCF under $z$-rotations by $\pi$, all solutions have shift-androtate $(x, y, z) \rightarrow\left(L_{x} / 2-x,-y, z+L_{z} / 2\right)$ symmetry, which turns fields $\left(h_{x}, h_{y}, h_{z}\right)$ into $\left(-h_{x},-h_{y}, h_{z}\right)$ (all transformations require appropriate $x$ and $z$ phase choices). The $\alpha$ dependence of the growth rates of several modes for the 
2D-3C configuration of Fig. 1 is plotted in Fig. 2 A $(y, z)$ cut through the marginal $(\mathrm{R}, \mathrm{S})$ mode is shown in Fig. 3

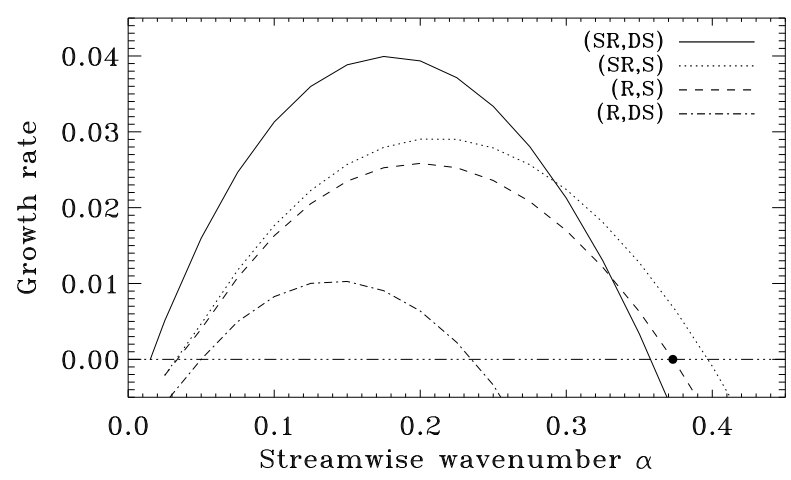

FIG. 2: Growth rates of the most unstable three-dimensional MRI eigenmodes for the 2D-3C configuration with $O(1)$ toroidal field shown in Fig. 1 as a function of the streamwise wavenumber $\alpha$. The legend indicates the symmetries of each mode.

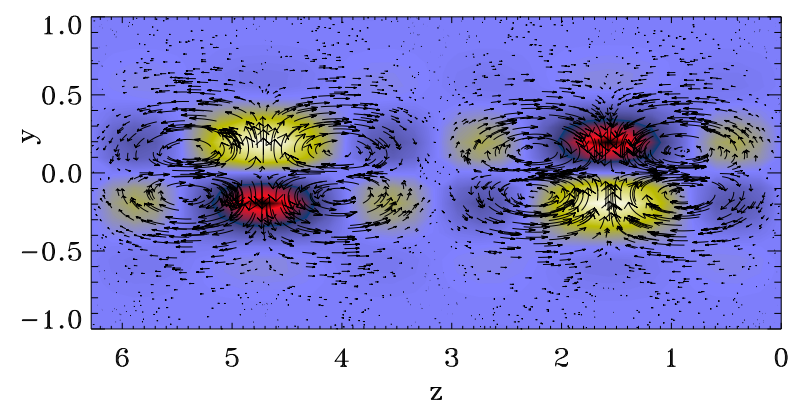

FIG. 3: Magnetic field in the $x=0$ plane of the near marginal $(\mathrm{R}, \mathrm{S}) \mathrm{MRI}$ eigenmode with $\alpha=0.375$, depicted by a black dot in Fig. 2 Same representation and parameters as in Fig. 1

In order to find a good initial guess to discover steady three-dimensional solutions with the Newton solver, one must select a marginally stable mode and check whether or not the $x$-averaged EMF due to its nonlinear interactions can take on the role of the artificial EMF (3) to sustain the poloidal field. This depends strongly on the selected MRI mode and on its streamwise wavenumber $\alpha$, much like in the hydrodynamic problem [12]. If the feedback is bad, the amplitude $A$ of the artificial EMF (and therefore the toroidal component of the $2 \mathrm{D}-3 \mathrm{C}$ field) is adjusted and a 2D-3C flow marginally unstable to a MRI mode with slightly different $\alpha$ is recalculated. This way, we identify a (R,S) MRI eigenmode (Fig. 3) whose nonlinear interactions create a toroidal EMF that has an interesting positive correlation with the artificial EMF (Fig. (4). Note that considering the feedback in the 2D-3C momentum equation is not important to find an interesting MRI mode since the goal is to replace an artificial forcing term imposed in the 2D-3C induction equation only. We final attempt a continuation with respect to the forcing amplitude

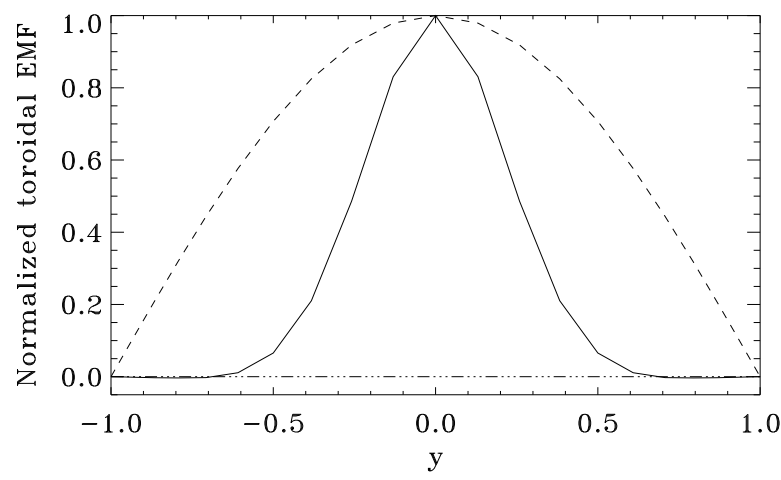

FIG. 4: Full line: normalized shearwise profile of the $k_{z}=\beta$ component of the toroidal EMF created by the self-interactions of the three-dimensional $\alpha=0.375$ MRI mode of Fig. 3 Dashed line: normalized shearwise profile of the artificial EMF 3 .

to find unforced $(A=0)$ solutions. The code is initialized with the 2D-3C base flow of Fig. 11 plus a small amount of the three-dimensional (R,S) MRI mode of Fig. 3, and $A$ is set to a slightly smaller value than that used to force the 2D-3C flow. The solver converges to a fully threedimensional forced solution, demonstrating that the bifurcation to three-dimensional solutions is subcritical with respect to $A$. Further confirmation of the crucial role of the MRI mode feedback is obtained by performing similar calculations initialized with marginal MRI modes with negative feedback (such as the (SR,DS) mode of Fig. 2 at $\alpha=0.355$ ), which reveal supercritical behaviour. The subcritical $(\mathrm{R}, \mathrm{S})$ branch can be followed down to $A=0$ (Fig. 5), at which point (black dot) nonlinear interactions due to the $x$-dependent part of the solution fully take over the forcing term. The $A=0$ point is therefore a fully three-dimensional subcritical nonlinear steady solution of the original MHD equations with no forcing (Fig. 6). The $x$-dependence of the solution, unlike for $A=1.5$, can no longer be described by a single Fourier mode.

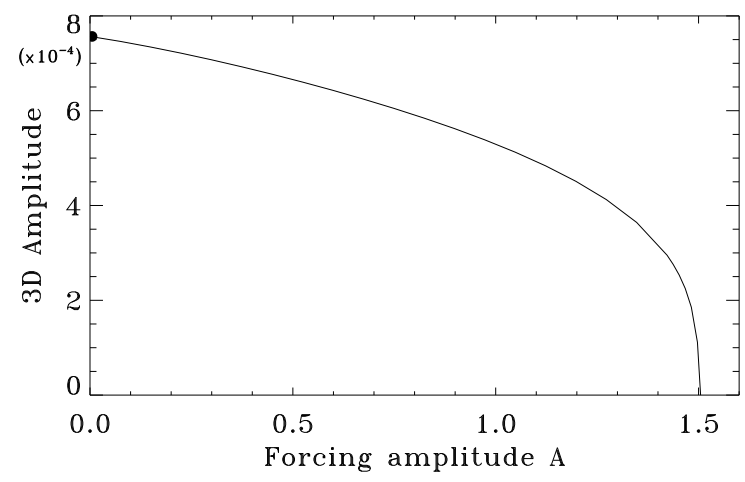

FIG. 5: $y$-integrated amplitude of the $k_{x}=0.375, k_{z}=0$ component of $v_{y}$ for three-dimensional steady forced solutions, as a function of the forcing amplitude $A$. $\left(N_{x}, N_{y}, N_{z}\right)=(12,32,32)$. 

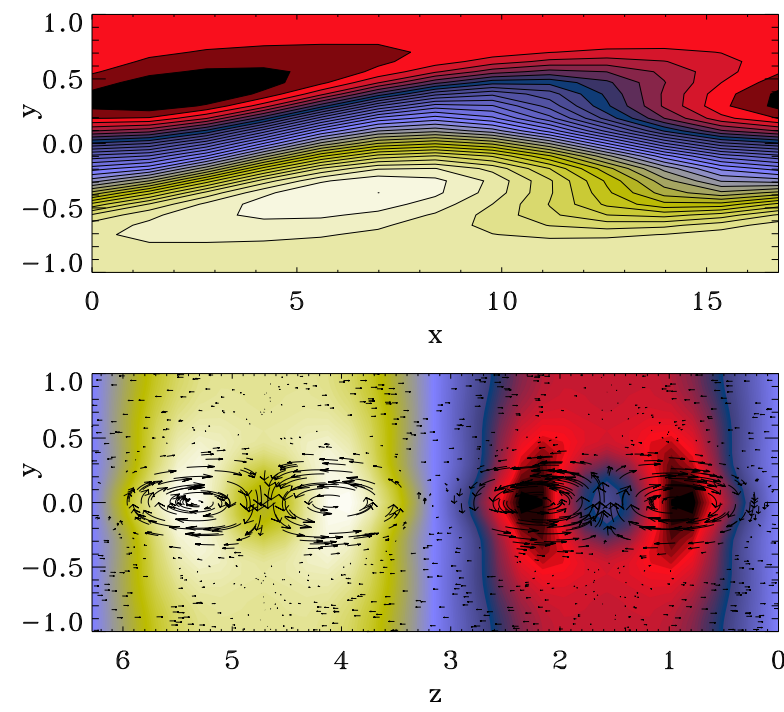

FIG. 6: Cuts through the unforced $(A=0)$ nonlinear steady solution of Fig. 5 ( $\alpha=0.375$, black dot). Top: $b_{z}$ at $z=L_{z} / 2$. Bottom: $b_{x}$ (color scale) and $\left(b_{y}, b_{z}\right)$ (arrows) at $x=L_{x} / 4$.

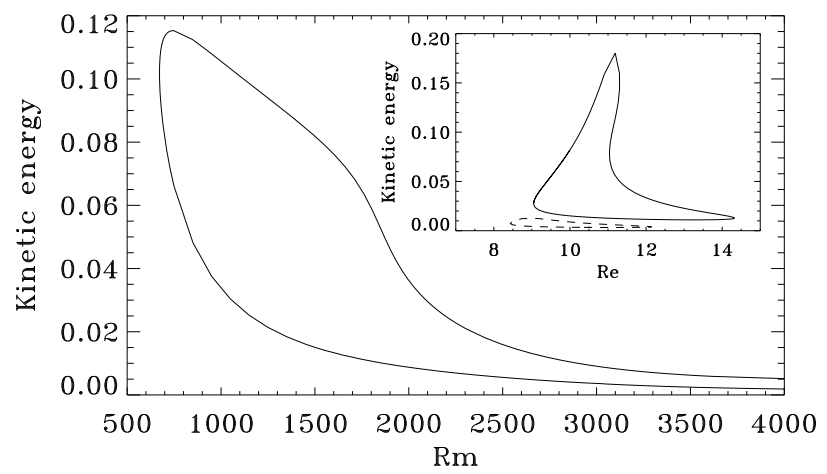

FIG. 7: Continuation with respect to $\operatorname{Rm}$ for $\operatorname{Re}=10,(\alpha, \beta)=$ $(0.375,1)$. Inset: continuation with respect to $\mathrm{Re}$ for $\mathrm{Rm}=1500$ (full line), $\mathrm{Rm}=3000$ (dashed line). $\left(N_{x}, N_{y}, N_{z}\right)=(8,24,32)$.

As shown in Fig. 7 this nonlinear steady solution can be continued to large Rm but exists only in a narrow range of Re. The reason for this could be that only at low Re do marginally unstable MRI modes of the toroidal magnetic field originate from a steady bifurcation. At larger Re, marginal MRI modes systematically arise from a Hopf bifurcation instead: pairs of steady eigenvalues corresponding to modes with the same symmetry collide and turn into complex-conjugate pairs for increasing Re. To discover similar dynamo solutions at larger Re, it may therefore prove necessary to consider time-dependent MRI modes instead of steady ones. This is unfortunately numerically far more challenging, since the symmetries used to reach decent three-dimensional resolutions are broken when the MRI modes turn into complex-conjugate pairs.

We have presented an instance of self-sustaining, nonlinear dynamo solution in Keplerian PCF, whose critical $\mathrm{Rm} \simeq 670$ is comparable to that found in zero-net-flux simulations (another Rm definition is used in [7]). Preliminary direct spectral numerical simulations seem to confirm independently the existence of this solution, whose structure is dominated by a coherent zero-net-flux toroidal magnetic field. We also discovered a (R,DS) branch, which makes it probable that many solutions with different symmetries exist. Such coherent structures are strictly speaking not turbulence but, like the hydrodynamics SSP, they probably act as organizing centers of the dynamics in phase space [10] and could play an important role in triggering and sustaining MHD turbulence in magnetized Keplerian disks, where all the basic ingredients of the dynamo are present. Hopefully, this idealized study will be helpful to uncover the details of the dynamo in more realistic set-ups.

We thank C. Cossu for his contribution to the code and A. Schekochihin and S. Fromang for fruitful discussions. We acknowledge support from the Leverhulme Trust and the Isaac Newton Trust.

* Electronic address: F.Rincon@ damtp.cam.ac.uk

[1] S. A. Balbus and J. F. Hawley, Rev. Mod. Phys. 70, 1 (1998).

[2] E. P. Velikhov, Sov. Phys. JETP 36, 1398 (1959); S. Chandrasekhar, Proc. Natl. Acad. Sci. 46, 253 (1960); S. A. Balbus and J. F. Hawley, ApJ 376, 214 (1991).

[3] J. F. Hawley, C. F. Gammie, and S. A. Balbus, ApJ 440, 742 (1995).

[4] J. Goodman and G. Xu, ApJ 432, 213 (1994).

[5] J.-F. Donati et al., Nature 438, 466 (2005).

[6] A. Brandenburg et al., ApJ 446, 741 (1995).

[7] T. P. Fleming, J. M. Stone, and J. F. Hawley, ApJ 530, 464 (2000).

[8] G. I. Ogilvie and J. E. Pringle, MNRAS 279, 152 (1996); C. Terquem and J. C. B. Papaloizou, MNRAS 279, 767 (1996).

[9] J. M. Hamilton, J. Kim, and F. Waleffe, J. Fluid Mech. 287, 317 (1995).

[10] F. Waleffe, Phys. Rev. Lett. 81, 4140 (1998); F. Waleffe, J. Fluid Mech. 435, 93 (2001); F. Waleffe, Phys. Fluids 15, 1517 (2003).

[11] M. T. Landahl, J. Fluid Mech. 98, 243 (1980).

[12] H. Wedin and R. R. Kerswell, J. Fluid Mech. 508, 333 (2004).

[13] F. Rincon, G. I. Ogilvie, and C. Cossu, A\&A 463, 817 (2007). 